\title{
Voronoi Diagram of Polygonal Chains Under the Discrete Fréchet Distance
}

\author{
Sergey Bereg * $\quad$ Marina Gavrilova ${ }^{\dagger} \quad$ Binhai Zhu ${ }^{\ddagger}$
}

\begin{abstract}
Polygonal chains are fundamental objects in many applications like pattern recognition and protein structure alignment. A well-known measure to characterize the similarity of two polygonal chains is the famous Fréchet distance. In this paper, for the first time, we consider the Voronoi diagram of polygonal chains in $d$-dimension $(d=2,3)$ under the discrete Fréchet distance. Given $n$ polygonal chains $\mathcal{C}$ in $d$-dimension $(d=2,3)$, each with at most $k$ vertices, we prove fundamental properties of such a Voronoi diagram $V D_{F}(\mathcal{C})$. Our main results are summarized as follows.

- The combinatorial complexity of $V D_{F}(\mathcal{C})$ is at least $\Omega\left(n^{\left\lfloor\frac{k+1}{2}\right\rfloor}\right)$; in fact, even a slice of $V D_{F}(\mathcal{C})$ could contain a $L_{\infty}$-metric Voronoi diagram in $k$ dimensions.

- The combinatorial complexity of $V D_{F}(\mathcal{C})$ is at most $O\left(n^{d k+\epsilon}\right)$, for $d=2,3$.

- Even if each polygonal chain degenerates into a point in three dimensions (3D), e.g., when we are given a set $P$ of $n$ co-planar points in 3D, the corresponding three-dimensional Voronoi diagram under the discrete Fréchet distance, $V D_{F}(P)$, has a combinatorial complexity of $\Omega\left(n^{2}\right)$.
\end{abstract}

Keywords: Voronoi diagram, Fréchet distance, discrete Fréchet distance, combinatorial complexity, protein structure alignment

\footnotetext{
${ }^{*}$ Department of Computer Science, University of Texas at Dallas, Richardson, TX 75083, USA. Email: besp@utdallas.edu.

${ }^{\dagger}$ Department of Computer Science, University of Calgary, Calgary, Alberta T2N 1N4, Canada. Email: marina@cpsc.ucalgary.ca.

${ }^{\ddagger}$ Department of Computer Science, Montana State University, Bozeman, MT 59717-3880, USA. Email: bhz@cs.montana.edu.
} 


\section{Introduction}

Fréchet distance was first defined by Maurice Fréchet in 1906 [8]. While known as a famous distance measure in the field of mathematics (more specifically, abstract spaces), it was Alt and Godau who first applied it in measuring the similarity of polygonal curves in 1992 [1]. In general, Fréchet distance between 2D polygonal chains (polylines) can be computed in polynomial time [1, 2], even under translation or rotation (though the running time is much higher) [3, 16]. While computing (approximating) Fréchet distance for surfaces is NP-hard [9], it is polynomially solvable for restricted surfaces [5].

In 1994, Eiter and Mannila defined the discrete Fréchet distance between two polygonal chains $A$ and $B$ and it turns out that this simplified distance is always realized by two vertices in $A$ and $B$ [7]. They also showed that with dynamic programming the discrete Fréchet distance between them can be computed in $O(|A||B|)$ time. (In fact, the dynamic programming algorithm works even when the vertices of the chains are in any fixed dimension.) In [10], Indyk defined a similar discrete Fréchet distance in some metric space and showed how to compute approximate nearest neighbors using that distance.

Recently, Jiang, Xu and Zhu applied the discrete Fréchet distance in aligning the backbones of proteins (which is called the protein structure-structure alignment problem) [11]. In fact, in this application the discrete Fréchet distance makes more sense as the backbone of a protein is simply a polygonal chain in $3 \mathrm{D}$, with each vertex being the alpha-carbon atom of a residue. So if the (continuous) Fréchet distance is realized by an alpha-carbon atom and some other point which does not represent an atom, it is not meaningful biologically. Jiang, et al. showed that given two planar polygonal chains the minimum discrete Fréchet distance between them, under both translation and rotation, can be computed in polynomial time. They also applied some ideas therein to design an efficient heuristic for the original protein structure-structure alignment problem in 3D and the empirical results showed that their alignment is more accurate compared with previously known solutions.

On the other hand, a lot is still unknown regarding discrete/continuous Fréchet distance. For instance, each Fréchet distance is a true distance measure, yet nobody has ever studied the Voronoi diagram under such an important distance measure. This problem is fundamental, it has potential applications, e.g., in protein structure alignment, especially with the ever increasing computational power. Imagine that we have some polylines $A_{1}, A_{2}, A_{3} \ldots$ in space. If we can construct the Voronoi diagram for $A_{1}, A_{2}, A_{3}, \ldots$ in space, then given a new polyline $B$ we can easily compute all the approximate alignment of $B$ with $A_{i}$ 's. The movement of $B$ defines a subspace (each point in the subspace represents a copy of $B$ ) and if we sample this subspace evenly then all we need to do is to locate all these sample points in the Voronoi diagram for $A_{i}$ 's.

Unfortunately, nothing is known about Voronoi diagram under the discrete/continuous Fréchet 
distance, even for the simplest case when each chain degenerates into a point in 3D. In this paper, we will present the first set of such results by proving some fundamental properties for both the general case and some special case. We believe that these results will be essential for us to design efficient algorithms for computing/approximating Voronoi diagram under the Fréchet distance. In this paper, all of our results are under the discrete Fréchet distance unless otherwise specified.

The paper is organized as follows. In Section 2, we introduce some basic definitions regarding Fréchet distance and review some known results. In Section 3, we show the combinatorial upper and lower bounds for the Voronoi diagram of a set of $2 \mathrm{D}$ polylines $\mathcal{C}, V D_{F}(\mathcal{C})$. In Section 4 , we sketch how to generalize the results in Section 3 to a set of 3D polylines. In Section 5, we show the quadratic lower bound for a special case where the $n$ polylines degenerate into $n$ co-planar points in 3D. In Section 6, we conclude the paper with several open problems.

\section{Preliminaries}

Given two polygonal chains $A, B$ with $|A|=k$ and $|B|=l$ vertices respectively, we aim at measuring the similarity of $A$ and $B$ (possibly under translation and rotation) such that their distance is minimized under certain measure. Among the various distance measures, the Hausdorff distance is known to be better suited for matching two point sets than for matching two polygonal chains; the (continuous) Fréchet distance is a superior measure for matching two polygonal chains, but it is not quite easy to compute [1].

Let $X$ be the Euclidean plane $\mathbb{R}^{2}$; let $d(a, b)$ denote the Euclidean distance between two points $a, b \in X$. The (continuous) Fréchet distance between two parametric curves $f:[0,1] \rightarrow X$ and $g:[0,1] \rightarrow X$ is

$$
\delta_{\mathcal{F}}(f, g)=\inf _{\alpha, \beta} \max _{s \in[0,1]} d(f(\alpha(s)), g(\beta(s))),
$$

where $\alpha$ and $\beta$ range over all continuous non-decreasing real functions with $\alpha(0)=\beta(0)=0$ and $\alpha(1)=\beta(1)=1$.

Imagine that a person and a dog walk along two different paths while connected by a leash; they always move forward, though at different paces. The minimum possible length of the leash is the Fréchet distance between the two paths. To compute the Fréchet distance between two polygonal curves $A$ and $B$ (in the Euclidean plane) of $|A|$ and $|B|$ vertices, respectively, Alt and Godau [1] presented an $O\left(|A||B| \log ^{2}(|A||B|)\right)$ time algorithm. Later this bound was reduced to $O(|A||B| \log (|A||B|))$ time [2].

We now define the discrete Fréchet distance following [7].

Definition 2.1 Given a polygonal chain (polyline) in the plane $P=<p_{1}, \ldots, p_{k}>$ of $k$ vertices, a $m$-walk along $P$ partitions the path into $m$ disjoint non-empty subchains $\left\{\mathcal{P}_{i}\right\}_{i=1 . . m}$ such that

\footnotetext{
${ }^{1}$ This definition holds in any fixed dimensions. In Section 4, we will cover the 3D case.
} 
$\mathcal{P}_{i}=<p_{k_{i-1}+1}, \ldots, p_{k_{i}}>$ and $0=k_{0}<k_{1}<\cdots<k_{m}=k$.

Given two planar polylines $A=\left\langle a_{1}, \ldots, a_{k}\right\rangle$ and $B=\left\langle b_{1}, \ldots, b_{l}\right\rangle$, a paired walk along $A$ and $B$ is a $m$-walk $\left\{\mathcal{A}_{i}\right\}_{i=1 . . m}$ along $A$ and a $m$-walk $\left\{\mathcal{B}_{i}\right\}_{i=1 . . m}$ along $B$ for some $m$, such that, for $1 \leq i \leq m,\left|\mathcal{A}_{i}\right|=1$ or $\left|\mathcal{B}_{i}\right|=1$ (that is, $\mathcal{A}_{i}$ or $\mathcal{B}_{i}$ contains exactly one vertex). The cost of a paired walk $W=\left\{\left(\mathcal{A}_{i}, \mathcal{B}_{i}\right)\right\}$ along two paths $A$ and $B$ is

$$
d_{F}^{W}(A, B)=\max _{i} \max _{(a, b) \in \mathcal{A}_{i} \times \mathcal{B}_{i}} d(a, b) .
$$

The discrete Fréchet distance between two polylines $A$ and $B$ is

$$
d_{F}(A, B)=\min _{W} d_{F}^{W}(A, B) .
$$

The paired walk that achieves the discrete Fréchet distance between two paths $A$ and $B$ is also called the Fréchet alignment of $A$ and $B$.

Consider the scenario in which the person walks along $A$ and the $\operatorname{dog}$ along $B$. Intuitively, the definition of the paired walk is based on three cases:

1. $\left|\mathcal{B}_{i}\right|>\left|\mathcal{A}_{i}\right|=1$ : the person stays and the dog moves forward;

2. $\left|\mathcal{A}_{i}\right|>\left|\mathcal{B}_{i}\right|=1$ : the person moves forward and the dog stays;

3. $\left|\mathcal{A}_{i}\right|=\left|\mathcal{B}_{i}\right|=1$ : both the person and the dog move forward.

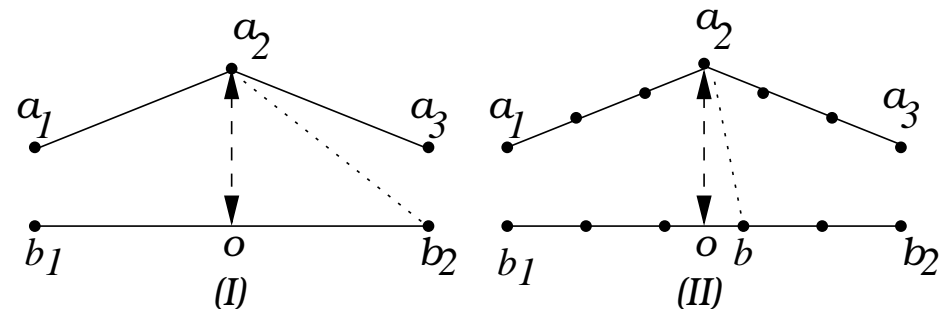

Fig. 1. The relationship between discrete and continuous Fréchet distances.

Eiter and Mannila presented a simple dynamic programming algorithm to compute $d_{F}(A, B)$ in $O(|A||B|)=O(k l)$ time [7]. Recently, Jiang et al. showed that the minimum discrete Fréchet distance between $A$ and $B$ under translation can be computed in $O\left(k^{3} l^{3} \log (k+l)\right)$ time, and under both translation and rotation it can be computed in $O\left(k^{4} l^{4} \log (k+l)\right)$ time [11]. They are significantly faster than the corresponding bounds for the continuous Fréchet distance. For continuous Fréchet distance, under translation, the current fastest algorithm for computing the minimum (continuous) Fréchet distance between $A, B$ takes $O\left((k l)^{3}(k+l)^{2} \log (k+l)\right)$ time [3]; under both translation and rotation, the bound is $O\left((k+l)^{11} \log (k+l)\right)$ time [16]. 
We comment that while the discrete Fréchet distance could be arbitrarily larger than the corresponding continuous Fréchet distance (e.g., in Fig. 1 (I), they are $d\left(a_{2}, b_{2}\right)$ and $d\left(a_{2}, o\right)$ respectively), by adding sample points on the polylines, one can easily obtain a close approximation of the continuous Fréchet distance using the discrete Fréchet distance (e.g., one can use $d\left(a_{2}, b\right)$ in Fig. 1 (II) to approximate $\left.d\left(a_{2}, o\right)\right)$. This fact was also pointed out by Indyk in [10]. Moreover, the discrete Fréchet distance is a more natural measure for matching the geometric shapes of biological sequences such as proteins. As we mentioned in the introduction, in such an application, continuous Fréchet does not make much sense to biologists.

In the remaining part of this paper, for the first time, we investigate the Voronoi diagram of a set of polygonal chains (polylines) in $d$-dimension, for $d=2,3$. While Voronoi diagram is a central structure in geometric computing and has been widely studied [13], it still attracts a lot of attention recently [4, 12] (including a new annual International Symposium on Voronoi Diagrams in Science and Engineering). We hope that our work will facilitate the understanding of the continuous and discrete Fréchet distance and further enable their applications in various areas, like pattern recognition and computational biology.

\section{The Combinatorial Complexity of $V D_{F}(\mathcal{C})$ in $2 \mathrm{D}$}

Let $A_{k}=<a_{1}, a_{2}, \ldots, a_{k}>$ and $B_{l}=<b_{1}, b_{2}, \ldots, b_{l}>$ be two polygonal chains in the plane where $a_{i}=\left(x\left(a_{i}\right), y\left(a_{i}\right)\right), b_{j}=\left(x\left(b_{j}\right), y\left(b_{j}\right)\right)$ and $k, l \geq 1$. We first have the following lemma, which is easy to prove.

Lemma 3.1 Let $A_{2}=<a_{1}, a_{2}>$ and $B_{2}=<b_{1}, b_{2}>$ be two line segments in the plane, then

$$
d_{F}\left(A_{2}, B_{2}\right)=\max \left(d\left(a_{1}, b_{1}\right), d\left(a_{2}, b_{2}\right)\right)
$$

For general polylines, we can generalize the above lemma as follows.

Lemma 3.2 Let $A_{k}=\left\langle a_{1}, a_{2}, \ldots, a_{k}>\right.$ and $B_{l}=\left\langle b_{1}, b_{2}, \ldots, b_{l}\right\rangle$ be two polygonal chains in the plane where $k, l \geq 1$. The discrete Fréchet distance between $A_{k}$ and $B_{l}$ can be computed as

$$
d_{F}\left(A_{k}, B_{l}\right)= \begin{cases}\max \left\{d\left(a_{i}, b_{1}\right), i=1,2, \ldots, k\right\} & \text { if } l=1, \\ \max \left\{d\left(a_{1}, b_{j}\right), j=1,2, \ldots, l\right\} & \text { if } k=1, \\ \max \left\{d\left(a_{k}, b_{l}\right), \min \left(d_{F}\left(A_{k-1}, B_{l-1}\right), d_{F}\left(A_{k}, B_{l-1}\right), d_{F}\left(A_{k-1}, B_{l}\right)\right)\right\} & \text { if } k, l>1 .\end{cases}
$$

Proof. As can be seen from Section 2, the discrete Fréchet distance can be computed using dynamic programming. We assume that the man walks along $A_{k}$ and the $\operatorname{dog}$ walks along $B_{l}$. If $l=1$ then the dog stays at $b_{1}$ and $d_{F}\left(A_{k}, B_{l}\right)=\max \left\{d\left(a_{i}, b_{1}\right), i=1,2, \ldots, k\right\}$. If $k=1$ then the man stays at $a_{1}$ and $d_{F}\left(A_{k}, B_{l}\right)=\max \left\{d\left(a_{1}, b_{j}\right), j=1,2, \ldots, l\right\}$. 
Suppose that both $k$ and $l$ are greater than 1 . If the man and dog both move in the last step then

$$
d_{F}\left(A_{k}, B_{l}\right)=\max \left(d\left(a_{k}, b_{l}\right), d_{F}\left(A_{k-1}, B_{l-1}\right)\right) .
$$

If only the man moves in the last step then

$$
d_{F}\left(A_{k}, B_{l}\right)=\max \left(d\left(a_{k}, b_{l}\right), d_{F}\left(A_{k-1}, B_{l}\right)\right)
$$

If only the dog moves in the last step then

$$
d_{F}\left(A_{k}, B_{l}\right)=\max \left(d\left(a_{k}, b_{l}\right), d_{F}\left(A_{k}, B_{l-1}\right)\right)
$$

The smallest value of (21),(3) and (41) is

$$
\max \left\{d\left(a_{k}, b_{l}\right), \min \left(d_{F}\left(A_{k-1}, B_{l-1}\right), d_{F}\left(A_{k}, B_{l-1}\right), d_{F}\left(A_{k-1}, B_{l}\right)\right)\right\}
$$

The lemma follows.

Based on the above lemma, we try to investigate the combinatorial complexity of $V D_{F}(\mathcal{C})$, the Voronoi diagram of a set $\mathcal{C}$ of $n$ planar polylines each with at most $k$ vertices. Following [6, 15], a Voronoi diagram is a minimization of distance functions to the sites (in this case the polylines in $\mathcal{C})$. We first quickly review a result on the upper bound of lower envelopes in high dimensions by Sharir [15].

Let $\Sigma=\left\{\sigma_{1}, \ldots, \sigma_{n}\right\}$ be a collection of $n(d-1)$-dimensional algebraic surface patches in $d$-space. Let $\mathcal{A}(\Sigma)$ be the arrangement of $\Sigma$. The result in [15] holds upon the following three conditions.

(i) Each $\sigma_{i}$ is monotone in the $x_{1} x_{2} \ldots x_{d-1}$-direction (i.e. any line parallel to $x_{d}$-axis intersects $\sigma_{i}$ in at most one point). Moreover, each $\sigma_{i}$ is a portion of a $(d-1)$-dimensional algebraic surface of constant maximum degree $b$.

(ii) The projection of $\sigma_{i}$ in $x_{d}$-direction onto the hyperplane $x_{d}=0$ is a semi-algebraic set defined in terms of a constant number of $(d-1)$-variate polynomials of constant maximum degree.

(iii) The surface patches $\sigma_{i}$ are in general position meaning that the coefficients of the polynomials defining surfaces and their boundaries are algebraically independent over rationals.

Theorem 3.1 [15] Let $\Sigma$ be a collection of $n(d-1)$-dimensional algebraic surface patches in $d$ space, which satisfy the above conditions (i),(ii), and (iii). Then the number of vertices of $\mathcal{A}(\Sigma)$ that lie at the lower envelope (i.e., level one) is $O\left(n^{d-1+\epsilon}\right)$, for any $\epsilon>0$.

We now try to show a general upper bound on the combinatorial complexity of $V D_{F}(\mathcal{C})$.

Polyline-point correspondence. Let $c_{1}, c_{2}, \ldots, c_{k}$ be the sequence of vertices of a polygonal chain $C$ and let $\left(x\left(c_{i}\right), y\left(c_{i}\right)\right)$ be the coordinates of vertex $c_{i}, i=1, \ldots, k$. Using this notation we view every polygonal chain $C$ with $k$ vertices in the plane as a point in $\mathbb{R}^{2 k}$ and vice versa. 
Lemma 3.3 Let $B \in \mathbb{R}^{2 l}$ be a polygonal chain of $l$ vertices $b_{1}, \ldots, b_{l}$ in the plane where $b_{i}=$ $\left(x\left(b_{i}\right), y\left(b_{i}\right)\right)$. Let $f: \mathbb{R}^{2 k} \rightarrow \mathbb{R}$ be the distance function defined as

$$
f(C)=d_{F}(C, B)
$$

where $C=\left(c_{1}, \ldots, c_{k}\right) \in \mathbb{R}^{2 k}$ is a polygonal chain with $k$ vertices and $c_{i}=\left(x\left(c_{i}\right), y\left(c_{i}\right)\right), i=1, \ldots, k$. The space $\mathbb{R}^{2 k}$ can be partitioned into at most $(k l)$ ! semi-algebraic sets $R_{1}, R_{2}, R_{3}, \ldots$ such that the function $f(C)$ with domain restricted to any $R_{i}$ is algebraic. Thus, the function $f(C)$ satisfies the above condition (i) and (ii).

Proof. We consider $\left(\begin{array}{l}k \\ 2\end{array}\right) \cdot\left(\begin{array}{l}l \\ 2\end{array}\right)$ manifolds in $\mathbb{R}^{2 k}$ defined as

$$
\left(x\left(c_{i}\right)-x\left(b_{j}\right)\right)^{2}+\left(y\left(c_{i}\right)-y\left(b_{j}\right)\right)^{2}=\left(x\left(c_{i^{\prime}}\right)-x\left(b_{j^{\prime}}\right)\right)^{2}+\left(y\left(c_{i^{\prime}}\right)-y\left(b_{j^{\prime}}\right)\right)^{2}
$$

for every four integer $i, i^{\prime}, j, j^{\prime}$ such that $1 \leq i<i^{\prime} \leq k$ and $1 \leq j<j^{\prime} \leq l$. They partition $\mathbb{R}^{2 k}$ into at most $(k l)$ ! semi-algebraic sets $R_{1}, R_{2}, R_{3}, \ldots$ corresponding to the order of distances between $c_{i}$ and $b_{j}$ for all $1 \leq i \leq k$ and $1 \leq j \leq l$.

Equation (1) in Lemma 3.2 implies that the function $f(C)$ restricted to a domain $R_{m}$ satisfies

$$
f(C)=\sqrt{\left(x\left(c_{i}\right)-x\left(b_{j}\right)\right)^{2}+\left(y\left(c_{i}\right)-y\left(b_{j}\right)\right)^{2}}
$$

for some pair $i, j$. The lemma follows.

We now prove the following theorem regarding the combinatorial upper bound for $V D_{F}(\mathcal{C})$.

Theorem 3.2 Let $\mathcal{C}$ be a collection of $n$ polygonal chains $C_{1}, \ldots, C_{n}$ each with at most $k$ vertices in the plane. The combinatorial complexity of the Voronoi diagram $\operatorname{VD}_{F}(\mathcal{C})$ is $O\left(n^{2 k+\epsilon}\right)$, for any $\epsilon>0$.

Proof. Let $c_{i 1}, c_{i 2}, \ldots, c_{i k}$ be the sequence of vertices of the chain $C_{i}, i=1, \ldots, n$ and let $\left(x\left(c_{i j}\right), y\left(c_{i j}\right)\right)$ be the coordinates of the vertex $c_{i j}, j=1, \ldots, k$. Using this notation we view every polygonal chain $C$ with $k$ vertices in the plane as a point in $\mathbb{R}^{2 k}$. The Voronoi diagram $V D_{F}(\mathcal{C})$ can be viewed as a diagram in $\mathbb{R}^{2 k}$. It is well-known that a Voronoi diagram can be interpreted as a minimization of distance functions to the sites [6, 15].

Let $f_{i}: \mathbb{R}^{2 k} \rightarrow \mathbb{R}, i=1, \ldots, n$, be the distance function defined as

$$
f_{i}(C)=d_{F}\left(C, C_{i}\right)
$$

where $C=\left(c_{1}, \ldots, c_{k}\right) \in \mathbb{R}^{2 k}$ is a polygonal chain with $k$ vertices and $c_{i}=\left(x\left(c_{i}\right), y\left(c_{i}\right)\right), i=1, \ldots, k$. By Lemma 3.3 the function $f_{i}(C)$ satisfies the conditions (i) and (ii). And condition (iii) is easily satisfied using an argument similar to [15]. The Voronoi diagram $V D_{F}(\mathcal{C})$ corresponds to the lower envelope in the arrangement of the surfaces $z=f_{i}(C)$ in $\mathbb{R}^{2 k+1}$. By Theorem 3.1 the combinatorial complexity of $V D_{F}(\mathcal{C})$ is $O\left(n^{2 k+\epsilon}\right)$. 
Combinatorial lower bound for $V D_{F}(\mathcal{C})$. We now present a general lower bound for $V D_{F}(\mathcal{C})$. In fact we show a much stronger result that even a slice of $V D_{F}(\mathcal{C})$ could contain a $L_{\infty}$-Voronoi diagram in $k$ dimensions, whose combinatorial complexity is $\Omega\left(n^{\left\lfloor\frac{k+1}{2}\right\rfloor}\right)$.

Schaudt and Drysdale proved that a $L_{\infty}$-Voronoi diagram in $k$ dimensions has combinatorial complexity of $\Omega\left(n^{\left\lfloor\frac{k+1}{2}\right\rfloor}\right)$ [14]. Let $S=\left\{p_{1}, p_{2}, \ldots, p_{n}\right\}$ be a set of $n$ points in $\mathbb{R}^{k}$ such that the $L_{\infty}$ Voronoi diagram of $S$ has complexity of $\Omega\left(n^{\left\lfloor\frac{k+1}{2}\right\rfloor}\right)$. Let $M>0$ be a real number such that the hypercube $[-M, M]^{k}$ contains $S$ and all the Voronoi vertices of the $L_{\infty}$-Voronoi diagram of $S$. We consider a $k$-dimensional flat $F$ of $\mathbb{R}^{2 k}$ defined as $F=\left\{\left(a_{1}, M, a_{2}, 2 M, \ldots, a_{k}, k M\right) \mid a_{1}, \ldots, a_{k} \in \mathbb{R}\right\}$ and a projection $\pi: F \rightarrow \mathbb{R}^{k}$ defined as $\pi(b)=\left(b_{1}, b_{3}, \ldots, b_{2 k-1}\right)$, for $b=\left(b_{1}, b_{2}, b_{3}, \ldots, b_{2 k-1}, b_{2 k}\right)$.

Let $\mathcal{C}=\left\{C_{1}, C_{2}, \ldots, C_{n}\right\}$, each $C_{i}$ being a planar polygonal chain with $k$ vertices. Let $C_{i}=$ $<c_{i 1}, c_{i 2}, \ldots, c_{i k}>$ and $c_{i m}=\left(x\left(c_{i m}\right), y\left(c_{i m}\right)\right)$, for $m=1,2, \ldots, k$. We set $c_{i m}=\left(p_{i m}, m M\right)$, for $1 \leq i \leq n, 1 \leq m \leq k$. Clearly, every $C_{i} \in F$. With $C_{i}$ we associate a point $C_{i}^{\prime}=\pi\left(C_{i}\right)$ in $\mathbb{R}^{k}$. We show that the intersection of $F$ and a $V D_{F}(\mathcal{C})$ has complexity of $\Omega\left(n^{\left\lfloor\frac{k+1}{2}\right\rfloor}\right)$.

Consider a point $T \in F$ such that $T^{\prime}=\pi(T)$ is a $L_{\infty}$-Voronoi vertex of $S$ in $\mathbb{R}^{k}$. Then $T^{\prime} \in[-M, M]^{k}$. At this point, the question is: what is the discrete Fréchet distance between $T$ and a chain $C_{i}$ ? Note that $d_{F}^{W}\left(T, C_{i}\right)<M$ if and only if $W=W_{0}$ where $W_{0}=\left\{\left(t_{m}, c_{i m}\right) \mid m=\right.$ $1, \ldots, k\}$. Therefore $d_{F}\left(T, C_{i}\right)=d_{F}^{W_{0}}\left(T, C_{i}\right)=\max \left\{\left|x\left(t_{1}\right)-x\left(c_{i 1}\right)\right|,\left|x\left(t_{2}\right)-x\left(c_{i 2}\right)\right|, \ldots, \mid x\left(t_{j}\right)-\right.$ $\left.x\left(c_{i j}\right)|, \ldots| x,\left(t_{k}\right)-x\left(c_{i k}\right) \mid\right\}$. This is exactly the $L_{\infty}$-distance between $T^{\prime}$ and $C_{i}^{\prime}$, or $d_{F}\left(T, C_{i}\right)=$ $d_{\infty}\left(T^{\prime}, C_{i}^{\prime}\right)$. Then the slice of $V D_{F}(\mathcal{C})$ contains the $L_{\infty}$-Voronoi diagram of $S$ in $k$ dimensions. We thus have the following theorem.

Theorem 3.3 The combinatorial complexity of $\mathrm{VD}_{F}(\mathcal{C})$ for a set $\mathcal{C}$ of $n$ planar polygonal chains with $k$ vertices is $\Omega\left(n^{\left\lfloor\frac{k+1}{2}\right\rfloor}\right)$; in fact even a $k$-dimensional slice of $\operatorname{VD}_{F}(\mathcal{C})$ can have a combinatorial complexity of $\Omega\left(n^{\left\lfloor\frac{k+1}{2}\right\rfloor}\right)$.

We comment that this lower bound is probably not tight and some significantly different method is needed to improve either the lower or upper bounds (or both). Nevertheless, the above theorems show the first non-trivial bounds for the size of $V D_{F}(\mathcal{C})$.

\section{The Combinatorial Complexity of $V D_{F}(\mathcal{C})$ in $3 \mathrm{D}$}

For protein-related applications, the input are polygonal chains in 3D. It turns out that Lemmas 3.1 and 3.2 hold for polygonal chains in $\mathbb{R}^{3}$. Similar to Lemma 3.3 we can prove

Lemma 4.1 Let $B \in \mathbb{R}^{3 l}$ be a polygonal chain of $l$ vertices $b_{1}, \ldots, b_{l}$ in $\mathbb{R}^{3}$, where $b_{i}=\left(x\left(b_{i}\right), y\left(b_{i}\right), z\left(b_{i}\right)\right)$. Let $f: \mathbb{R}^{3 k} \rightarrow \mathbb{R}$ be the distance function defined as

$$
f(C)=d_{F}(C, B)
$$


where $C=\left(c_{1}, \ldots, c_{k}\right) \in \mathbb{R}^{3 k}$ is a polygonal chain with $k$ vertices and $c_{i}=\left(x\left(c_{i}\right), y\left(c_{i}\right), z\left(c_{i}\right)\right), i=$ $1, \ldots, k$. The space $\mathbb{R}^{3 k}$ can be partitioned into at most $(k l)$ ! semi-algebraic sets $R_{1}, R_{2}, R_{3}, \ldots$ such that the function $f(C)$ with domain restricted to any $R_{i}$ is algebraic. Thus, the function $f(C)$ satisfies the above condition (i) and (ii).

The main difference is that the manifolds in $\mathbb{R}^{3 k}$ are defined as

$$
\left(x\left(c_{i}\right)-x\left(b_{j}\right)\right)^{2}+\left(y\left(c_{i}\right)-y\left(b_{j}\right)\right)^{2}+\left(z\left(c_{i}\right)-z\left(b_{j}\right)\right)^{2}=\left(x\left(c_{i^{\prime}}\right)-x\left(b_{j^{\prime}}\right)\right)^{2}+\left(y\left(c_{i^{\prime}}\right)-y\left(b_{j^{\prime}}\right)\right)^{2}+\left(z\left(c_{i^{\prime}}\right)-z\left(b_{j^{\prime}}\right)\right)^{2} .
$$

Using Lemma 4.1 we can prove similar to Theorem 3.2 the following bound.

Theorem 4.1 Let $\mathcal{C}$ be a collection of $n$ polygonal chains $C_{1}, \ldots, C_{n}$ each with at most $k$ vertices in $\mathbb{R}^{3}$. The combinatorial complexity of the Voronoi diagram $\operatorname{VD}_{F}(\mathcal{C})$ is $O\left(n^{3 k+\epsilon}\right)$, for any $\epsilon>0$.

We comment that the lower bound in Theorem 3.3 is in fact a special case for $V D_{F}(\mathcal{C})$ in $\mathbb{R}^{3}$.

\section{Lower Bound for a Simple Degenerate Case}

In this section, we present a lower bound for a simple degenerate case. Under this degenerate case, the geometric bisectors are easy to visualize. Nevertheless, we will see that Voronoi diagram under the discrete (and continuous) Fréchet distance is much more complex compared with the corresponding Euclidean case. In fact, for the same construction the former Voronoi diagram has a combinatorial complexity of $\Omega\left(n^{2}\right)$ while the latter Voronoi diagram (under the Euclidean metric) is only of size $\Theta(n)$.

First, let all the polygonal chains be $2 \mathrm{D}$ line segments, i.e., $\mathcal{C}=\left\{C_{i} \mid C_{i}=\left\langle a_{i}, b_{i}\right\rangle, a_{i}=\right.$ $\left(a_{i 1}, a_{i 2}\right), b_{i}=\left(b_{i 1}, b_{i 2}\right)$, for $\left.1 \leq i \leq n\right\}$. Then we set $b_{i 2}=0$ for all $i$. As a matter of fact, $\mathcal{C}$ is equivalent to a set $P$ of $n$ points in 3 D, i.e., $P=\left\{\left(a_{i 1}, a_{i 2}, b_{i 1}\right) \mid 1 \leq i \leq n\right\}$.

We now further construct points in $P$ in two equal parts, i.e., $P=P_{1} \cup Q_{1}$, with $P_{1}=\left\{p_{i}=\right.$ $(0,0, i) \mid 1 \leq i \leq n / 2\}$ and $Q_{1}=\{(j, 0,0) \mid 1 \leq j \leq n / 2\}$ (without loss of generality, assume that $n$ is even).

Let us look at the bisector between $p_{i}$ and $p_{i+1}$. Be reminded that $p_{i}$ and $p_{i+1}$ are in fact line segments; i.e., $p_{i}=\langle(0,0),(i, 0)\rangle$ and $p_{i+1}=\langle(0,0),(i+1,0)\rangle$. Let $s=(x, y, z, 0)$ be a point on the bisector of $p_{i}$ and $p_{i+1}$ in $3 \mathrm{D}$. Then the discrete Fréchet distance $d_{F}\left(p_{i}, s\right)$ is $d_{F}\left(p_{i}, s\right)=\max \left\{|z-i|, \sqrt{x^{2}+y^{2}}\right\}$. Similarly, $d_{F}\left(p_{i+1}, s\right)=\max \left\{|z-i-1|, \sqrt{x^{2}+y^{2}}\right\}$.

So, what does this bisector look like? When $|z-i|$ and $|z-i-1|$ are bigger than $\sqrt{x^{2}+y^{2}}$, then the bisector is a plane $z=\frac{2 i+1}{2}$; when $\sqrt{x^{2}+y^{2}}$ is bigger the bisector are two paraboloids $(z-i)^{2}=x^{2}+y^{2}$ and $(z-i-1)^{2}=x^{2}+y^{2}$. The intersection of the plane $z=\frac{2 i+1}{2}$ and these two paraboloids is, of course, a circle $x^{2}+y^{2}=\left(\frac{1}{2}\right)^{2}$ (on the plane $z=\frac{2 i+1}{2}$ ). See Fig. 2 (part I). 


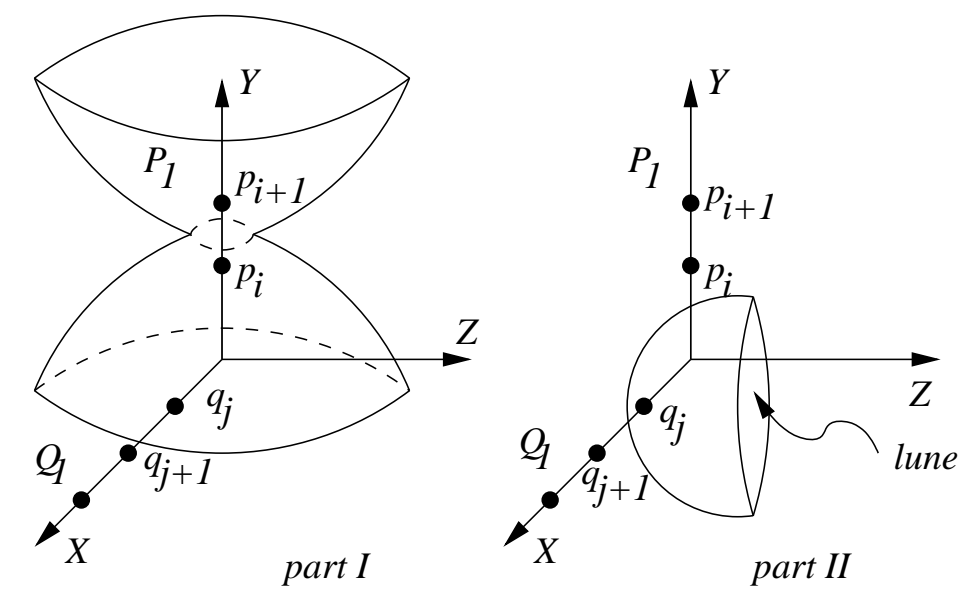

Fig. 2. Bisectors for $p_{i}, p_{i+1}$ and for $q_{j}, q_{j+1}$.

Next let us look at the bisector between $q_{j}$ and $q_{j+1}$. Again, be reminded that $q_{j}$ and $q_{j+1}$ are in fact line segments; i.e., $q_{j}=\left\langle(j, 0),(0,0)>\right.$ and $q_{j+1}=\langle(j+1,0),(0,0)\rangle$. For a point $s=(x, y, z)$ on the bisector of $q_{j}$ and $q_{j+1}$ in 3D, we have the discrete Fréchet distance $d_{F}\left(q_{j}, s\right)=\max \left\{|z|, \sqrt{(x-j)^{2}+y^{2}}\right\}$ and $d_{F}\left(q_{j+1}, s\right)=\max \left\{|z|, \sqrt{(x-j-1)^{2}+y^{2}}\right\}$.

In this case, the bisector is even more interesting (and complex): When $|z|$ is bigger, the bisector contains all the points satisfying $z^{2} \geq(x-j)^{2}+y^{2}$ and $z^{2} \geq(x-j-1)^{2}+y^{2}$ and this is not even a manifold. Geometrically, this is the intersection of the two paraboloids $z^{2} \geq(x-j)^{2}+y^{2}$ and $z^{2} \geq(x-j-1)^{2}+y^{2}$ and on each vertical slice $z=c$, for some constant $c$, this is a lune determined by two disks $(x-j)^{2}+y^{2} \leq c^{2}$ and $(x-j-1)^{2}+y^{2} \leq c^{2}$. Of course, when $|z|$ is smaller, the bisector is exactly the plane $x=\frac{2 j+1}{2}$. See Fig. 2 (part II).

So how do we determine the combinatorial complexity of $V D_{F}\left(P_{1} \cup Q_{1}\right)$ ? In this case, the Voronoi vertex is not a geometric point. We prove below that we can take the first intersection of the bisector of $p_{i}, p_{i+1}$ and the bisector of $q_{j}, q_{j+1}$, which is a geometric point, and then show that this intersection point is farther to some points in $P_{1}-\left\{p_{i}, p_{i+1}\right\}$ and $Q_{1}-\left\{q_{j}, q_{j+1}\right\}$ than to $p_{i}, p_{i+1}, q_{j}, q_{j+1}$ and it is never closer to any point in $P_{1}-\left\{p_{i}, p_{i+1}\right\}$ and $Q_{1}-\left\{q_{j}, q_{j+1}\right\}$ than to $p_{i}, p_{i+1}, q_{j}, q_{j+1}$. Then this point contributes an $\Omega(1)$ cost to the combinatorial complexity of $V D_{F}\left(P_{1} \cup Q_{1}\right)$. Finally we show that there are $\Omega(n)$ such $p_{i}, p_{i+1}$ pairs and $\Omega(n)$ such $q_{j}, q_{j+1}$ pairs. And this will conclude the proof that $V D_{F}(P)$ has a combinatorial complexity of $\Omega\left(n^{2}\right)$, or, put it in another way, given $n$ co-planar points in $3 \mathrm{D}$ the Voronoi diagram under the discrete Fréchet distance has a combinatorial complexity of $\Omega\left(n^{2}\right)$. This is significantly different from the corresponding Euclidean Voronoi diagram of $n$ co-planar points, has a combinatorial complexity of $\Theta(n)[13]$.

Now let us finish the technical details. The first intersection of the bisector of $p_{i}, p_{i+1}$ and the bisector of $q_{j}, q_{j+1}$ is determined by $z^{2}=(x-j-1)^{2}+y^{2}$ and $(z-i)^{2}=x^{2}+y^{2}$. Therefore, we have 
the intersection point $t_{i j}=\left(\frac{j+1}{2}, \frac{\sqrt{i^{2}-(j+1)^{2}}}{2}, \frac{i}{2}\right)$. Clearly, if two pairs $(i, j)$ and $\left(i^{\prime}, j^{\prime}\right)$ are different then $t_{i j} \neq t_{i^{\prime} j^{\prime}}$. We now show that $t_{i j}$ is never closer to points in $P_{1}-\left\{p_{i}, p_{i+1}\right\}$ and $Q_{1}-\left\{q_{j}, q_{j+1}\right\}$ than to $p_{i}, p_{i+1}, q_{j}, q_{j+1}$ (and $t_{i j}$ is farther to some points in $P_{1}-\left\{p_{i}, p_{i+1}\right\}$ and $Q_{1}-\left\{q_{j}, q_{j+1}\right\}$ than to $\left.p_{i}, p_{i+1}, q_{j}, q_{j+1}\right)$. This can be done by showing $d_{F}\left(t_{i j}, p_{h}\right)>\frac{i}{2}$ for $h>i$ and $d_{F}\left(t_{i j}, p_{h}\right)=\frac{i}{2}$ for $h<i$; and $d_{F}\left(t_{i j}, q_{m}\right)>\frac{i}{2}$ for $m>j+1$ and $d_{F}\left(t_{i j}, q_{k}\right)=\frac{i}{2}$ for $m<j+1$. We have

$$
d_{F}\left(t_{i j}, p_{h}\right)=\max \left\{\sqrt{\left(\frac{j+1}{2}\right)^{2}+\frac{i^{2}-(j+1)^{2}}{4}},\left|\frac{i}{2}-h\right|\right\},
$$

which is $d_{F}\left(t_{i j}, p_{h}\right)=\max \left\{\frac{i}{2},\left|\frac{i}{2}-h\right|\right\}>\frac{i}{2}$, when $h>i$ and $d_{F}\left(t_{i j}, p_{h}\right)=\max \left\{\frac{i}{2},\left|\frac{i}{2}-h\right|\right\}=\frac{i}{2}$, when $h<i$. Similarly,

$$
d_{F}\left(t_{i j}, q_{m}\right)=\max \left\{\sqrt{\left(\frac{j+1}{2}-m\right)^{2}+\frac{i^{2}-(j+1)^{2}}{4}},\left|\frac{i}{2}\right|\right\},
$$

which is $d_{F}\left(t_{i j}, q_{m}\right)=\max \left\{\frac{i^{2}-4 m(j+1)+4 m^{2}}{2}, \frac{i}{2}\right\}>\frac{i}{2}$, when $m>j+1$ and $d_{F}\left(t_{i j}, q_{m}\right)=\max \left\{\frac{i^{2}-4 m(j+1)+4 m^{2}}{2}, \frac{i}{2}\right\}>\frac{i}{2}$, when $m<j+1$. Be reminded that if two pairs $(i, j)$ and $\left(i^{\prime}, j^{\prime}\right)$ are different then $t_{i j} \neq t_{i^{\prime} j^{\prime}}$. Consequently, each bisector of $p_{i}, p_{i+1}$ and each bisector of $q_{j}, q_{j+1}$ determines a distinct Voronoi point (on a Voronoi vertex) which is never closer to points in $P_{1}-\left\{p_{i}, p_{i+1}\right\}$ and $Q_{1}-\left\{q_{j}, q_{j+1}\right\}$ than to $p_{i}, p_{i+1}, q_{j}, q_{j+1}$ and is farther to some points in $P_{1}-\left\{p_{i}, p_{i+1}\right\}$ and $Q_{1}-\left\{q_{j}, q_{j+1}\right\}$ than to $p_{i}, p_{i+1}, q_{j}, q_{j+1}$. We thus have the following theorem.

Theorem 5.1 The combinatorial complexity of $\mathrm{VD}_{F}(P)$ for a set $P$ of $n$ degenerate planar line segments, which is a set of $n$ co-planar points in $3 D$, is $\Omega\left(n^{2}\right)$.

Since the continuous and the discrete Fréchet distance are identical for line segments, we also have the following corollary.

Corollary 5.1 The combinatorial complexity of the Voronoi diagram for a set $P$ of $n$ degenerate planar line segments (which is a set of $n$ co-planar points in 3D) under the continuous Fréchet distance measure is $\Omega\left(n^{2}\right)$.

\section{Concluding Remarks}

In this paper, for the first time, we study the Voronoi diagram of polylines in 2D and 3D under the discrete Fréchet distance. We show combinatorial upper and lower bounds for such a Voronoi diagram. At this point, to make the diagram useful, we need to present efficient (approximation) algorithms to construct such a Voronoi diagram for decent $k$ (say $k=10 \sim 20$ ) so that one can first use a $(k-1)$-link chain to approximate a general input polyline. Although the running time might still look too high (due to the $O(k)$ exponent in the running time), the good news is that in many applications like protein structural alignment, $n$ is not very large. Another open problem, obviously, is to improve the combinatorial bounds shown in this paper. 


\section{Acknowledgment}

We thank Minghui Jiang for pointing out the gap in an earlier version of the arguments for Theorem 3.3 .

\section{References}

[1] H. Alt and M. Godau. Measuring the resemblance of polygonal curves. In Proceedings of the 8th Annual Symposium on Computational Geometry (SoCG'92), pages 102-109, 1992.

[2] H. Alt and M. Godau. Computing the Fréchet distance between two polygonal curves. Intl. J. Computational Geometry and Applications, 5:75-91, 1995.

[3] H. Alt, C. Knauer and C. Wenk. Matching polygonal curves with respect to the Fréchet distance. In Proceedings of the 18th Annual Symposium on Theoretical Aspects of Computer Science (STACS'01), pages 63-74, 2001.

[4] T. Asano, J. Matousek and T. Tokuyama. The distance trisector curve. In Proceedings of the 38th Annual Symposium on Theory of Comput.(STOC'06), pages 336-343, 2006.

[5] K. Buchin, M. Buchin and C. Wenk. Computing the Fréchet distance between simple polygons in polynomial time. In Proceedings of the 22nd Annual Symposium on Computational Geometry (SoCG'06), pages 80-87, 2006.

[6] H. Edelsbrunner and R. Seidel. Voronoi diagrams and arrangements. Discrete Comput. Geom., 1:25-44, 1986.

[7] T. Eiter and H. Mannila. Computing discrete Fréchet distance. Tech. Report CD-TR 94/64, Information Systems Department, Technical University of Vienna, 1994.

[8] M. Fréchet. Sur quelques points du calcul fonctionnel. Rendiconti del Circolo Mathematico di Palermo, 22:1-74, 1906.

[9] M. Godau. On the complexity of measuring the similarity between geometric objects in higher dimensions. PhD thesis, Freie Universitaet Berlin, 1998.

[10] P. Indyk. Approximate nearest neighbor algorithms for Fréchet distance via product metrics. In Proceedings of the 18th Annual Symposium on Computational Geometry (SoCG'02), pages 102-106, 2002.

[11] M. Jiang, Y. Xu and B. Zhu. Protein structure-structure alignment with discrete Fréchet distance. In Proceedings of the 5th Asia-Pacific Bioinformatics Conf. (APBC'07), pages 131$141,2007$. 
[12] F. Nielsen, J.-D. Boissonnat, and R. Nock. On Bregman Voronoi diagrams. In Proc. 18th ACM-SIAM Sympos. Discrete Algorithms, pages 746-755, 2007.

[13] F.P. Preparata and M.I. Shamos. Computational Geometry: An Introduction. Springer-Verlag, 1985.

[14] B. Schaudt and S. Drysdale. Higher dimensional Delaunay diagrams for convex distance functions. In Proceedings of the 4 th Canadian Conf. Comp. Geom. (CCCG'92), pages 274-279, 1992.

[15] M. Sharir. Almost tight upper bounds for lower envelopes in higher dimensions. Discrete Comp. Geom., 12:327-345, 1994.

[16] C. Wenk. Shape Matching in Higher Dimensions. PhD thesis, Freie Universitaet Berlin, 2002. 